Research Paper

\title{
The potential impact of the COVID-19 pandemic on the tuberculosis epidemic a modelling analysis
}

\author{
Lucia Cilloni $^{\mathrm{a}, 1}$, Han Fu ${ }^{\mathrm{a}, 1}$, Juan F Vesga ${ }^{\mathrm{a}}$, David Dowdy ${ }^{\mathrm{b}}$, Carel Pretorius ${ }^{\mathrm{c}}$, Sevim Ahmedov ${ }^{\mathrm{d}}$, \\ Sreenivas A. Nair ${ }^{\mathrm{e}}$, Andrei Mosneaga ${ }^{\mathrm{e}}$, Enos Masini ${ }^{\mathrm{e}}$, Suvanand Sahu ${ }^{\mathrm{e}}$, \\ Nimalan Arinaminpathy, ${ }^{\mathrm{e}, *}$ \\ ${ }^{a}$ MRC Centre for Global Infectious Disease Analysis, School of Public Health, Imperial College London, United Kingdom \\ ${ }^{\mathrm{b}}$ Department of Epidemiology, Johns Hopkins Bloomberg School of Public Health, Baltimore, MD, USA \\ ${ }^{c}$ Avenir Health, Glastonbury, Connecticut, USA \\ d Bureau for Global Health, Infectious Diseases Division, United States Agency for International Development, Washington, DC, USA \\ e Stop TB Partnership, Geneva, Switzerland
}

\section{A R T I C L E I N F O}

\section{Article History:}

Received 3 August 2020

Revised 25 September 2020

Accepted 5 October 2020

Available online 24 October 2020

\section{Keywords:}

Tuberculosis

Covid-19

Epidemiology

Mathematical modellingabstract

\begin{abstract}
A B S T R A C T
Background: Routine services for tuberculosis (TB) are being disrupted by stringent lockdowns against the novel SARS-CoV-2 virus. We sought to estimate the potential long-term epidemiological impact of such disruptions on TB burden in high-burden countries, and how this negative impact could be mitigated.

Methods: We adapted mathematical models of TB transmission in three high-burden countries (India, Kenya and Ukraine) to incorporate lockdown-associated disruptions in the TB care cascade. The anticipated level of disruption reflected consensus from a rapid expert consultation. We modelled the impact of these disruptions on TB incidence and mortality over the next five years, and also considered potential interventions to curtail this impact.

Findings: Even temporary disruptions can cause long-term increases in TB incidence and mortality. If lockdown-related disruptions cause a temporary 50\% reduction in TB transmission, we estimated that a 3-month suspension of TB services, followed by 10 months to restore to normal, would cause, over the next 5 years, an additional 1.19 million TB cases ( $\mathrm{Crl} 1.06-1.33)$ and 361,000 TB deaths ( $\mathrm{CrI} 333-394$ thousand) in India, 24,700 (16,100-44,700) TB cases and 12,500 deaths (8.8-17.8 thousand) in Kenya, and 4,350 (826-6,540) cases and 1,340 deaths (815-1,980) in Ukraine. The principal driver of these adverse impacts is the accumulation of undetected TB during a lockdown. We demonstrate how long term increases in TB burden could be averted in the short term through supplementary "catch-up" TB case detection and treatment, once restrictions are eased.

Interpretation: Lockdown-related disruptions can cause long-lasting increases in TB burden, but these negative effects can be mitigated with rapid restoration of TB services, and targeted interventions that are implemented as soon as restrictions are lifted.

Funding: USAID and Stop TB Partnership
\end{abstract}

(C) 2020 The Authors. Published by Elsevier Ltd. This is an open access article under the CC BY-NC-ND license (http://creativecommons.org/licenses/by-nc-nd/4.0/)

\section{Introduction}

The emergence of the novel virus SARS-CoV-2 has caused morbidity, mortality and societal disruption on a global scale. In the absence of pharmaceutical interventions, many countries have resorted to population-wide lockdowns to slow the spread of the virus and to allow their health systems to cope [1]. These lockdowns have had an

\footnotetext{
* Corresponding author.

E-mail address: nim.pathy@imperial.ac.uk (N. Arinaminpathy).

1 Contributed equally
}

important effect on SARS-CoV-2 transmission [2,3]. However, unintended consequences are inevitable with such sweeping measures. In low- and middle-income countries with health systems already under strain, the adverse effects of disruptions in health services (for example, ongoing transmission of infectious diseases) can last far beyond the period of the disruptions themselves [4-6].

In the present study we focus on tuberculosis (TB) - globally, the leading cause of death due to an infectious disease [7]. In recent decades TB incidence and mortality have been steadily declining, reflecting ongoing improvements in diagnosis, treatment and prevention [8]. However, in March 2020 a rapid analysis conducted by the Stop 


\section{Research in context}

\section{Evidence before this study}

We searched both the published and grey literature for relevant studies in this context, using the terms "(SARS-CoV-2 OR coronavirus OR COVID-19) AND Tuberculosis," limited to publications in English. One modelling study found it unlikely that TB transmission reductions could compensate for the adverse impacts of disrupted TB services. Another study used approaches relating notifications to TB mortality, to estimate the excess TB deaths that could occur globally, under different lockdown scenarios. We found no studies examining the potential impact of disruptions at different stages of the TB care cascade, nor for post-lockdown strategies for reconstituting TB services.

\section{Added value of this study}

We focused on India, Kenya and Ukraine, three high-burden countries, representing contrasting epidemiological conditions. In each country we modelled the impact of a range of lockdown scenarios, informed by expert consensus on the potential effects of a lockdown at various points in the TB care cascade. For each country we additionally examined which type of disruption (i.e. acting at which point on the care cascade) would be most associated with excess TB burden. Using insights from this analysis, we identified potential strategies for high-burden countries to bring their TB responses back on track.

\section{Implications of all the available evidence}

Even short-term disruptions can trigger escalations in TB burden that can take years to return to pre-lockdown levels. Such increases can be explained by an accumulating pool of undetected, untreated TB during a lockdown. Accordingly, country preparedness should involve 'catch-up' plans to intensify screening, evaluation and treatment of 'missed' cases of TB, immediately upon lifting a lockdown.

TB Partnership brought attention to severe impacts of COVID-related lockdowns on TB care in different countries [9]. For example, in the weeks following the imposition of a nationwide lockdown on March 24,2020 , India reported an $80 \%$ drop in daily notifications of TB [9] relative to average pre-lockdown levels. Similar changes have been reported elsewhere, for example with South Africa reporting a drop in numbers tested for TB by almost a half [10]. Such declines may be partly due to delays in reporting but are also likely to reflect reductions in access to diagnosis and treatment, potentially having a lasting impact on TB burden at a country-wide level. Missed diagnoses would mean increased opportunities for transmission, while worsened treatment outcomes increase the risk of death from TB. Therefore, while lockdowns are an important measure to mitigate the immediate impact of COVID-19, it is critical to anticipate (i) the potential long-term impact of these measures on TB and other diseases, and (ii) how this impact might be stemmed, in the short term, by appropriately targeted investment and effort. We therefore aimed to examine these questions using mathematical modelling of $\mathrm{TB}$ transmission dynamics. Building on earlier modelling conducted for the 2019 Lancet Commission on Tuberculosis [11,12], we modelled the potential TB-related impact of COVID-related lockdowns - and mitigating effects of potential post-lockdown interventions - in three focal countries: India, the Republic of Kenya, and Ukraine. It is not possible to predict the extent or depth of lockdowns in any given country, let alone their impact on TB services. Therefore, the primary purpose of our analysis is not to predict future TB trajectories, but rather to identify the critical drivers through which a lockdown can lead to elevated TB incidence and mortality, and to apply these insights to understand how best mitigate the long-term adverse impact of any lockdown-related disruptions.

\section{Methods}

\section{Model overview}

For each country we drew from previously published models of $\mathrm{M}$. tuberculosis transmission [12], which were designed to capture essential features of the TB care cascade. For the current analysis, this approach allowed us to model the impact of disruptions acting at multiple points in the care cascade. The three country settings offer examples of contrasting types of TB care cascade: for India we incorporated the dominant role of the private healthcare sector in providing TB care;[13] for Kenya, the role of HIV in driving TB dynamics; [14] and for Ukraine, the burden of drug resistance [15]. The overall model structure is illustrated schematically in Fig. S1 in the appendix. As noted above, the purpose of the analysis was not to predict TB trajectories in these three countries, but rather to examine the importance of different types of disruptions (i.e. acting at different stages of the TB care cascade), thus providing insights that could be relevant to other high-burden countries sharing similar characteristics. We calibrated each country model to the available data on TB burden, including WHO estimates of TB incidence and mortality [7], and on the burden of drug resistance (see Table S1). Full details of each model are provided in the Supporting Information.

Calibration was performed using Markov Chain Monte Carlo (MCMC) simulation [16-18], whereby we allowed model parameters to vary over pre-specified prior distributions, using a likelihood function based on the calibration targets listed above to weight simulations according to their fit to the observed data. For each country, we drew 250 samples from the weighted (posterior) density of simulations following burn-in and thinning as described in the Supporting Information. We then performed model projections on the basis of each of these samples, under the lockdown scenarios described below. For any model projection (for example, incidence over time), we estimated Bayesian credible intervals as $2.5^{\text {th }}$ and $97.5^{\text {th }}$ percentiles, and central estimates as $50^{\text {th }}$ percentiles, of the corresponding posterior density.

\section{Modelling the impact of disruptions}

Disruptions to TB services can act at all stages of the TB care cascade. During a lockdown, movement restrictions would curtail opportunities for those experiencing TB symptoms to seek care. Even once these people are able to visit a provider or health facility, the diagnostic and laboratory capacity needed to support TB diagnosis may be severely reduced - for example, with molecular diagnostic tools for TB being repurposed for COVID-19 [19] or TB laboratory staff being redirected to Covid-19 efforts. National TB programmes are investing significant effort to continue supporting those already on TB treatment, but there are also concerns that lockdown conditions may interfere with the continued supply of drugs [20]. To capture this range of possible disruptions, we performed a rapid consultation amongst experts at the Stop TB Partnership and the United States Agency for International Development (USAID). Table 1 lists those experts' consensus opinion as to the degree to which TB services could be disrupted by COVIDrelated lockdowns, at each step of the care cascade. There is substantial uncertainty around these possible impacts, and as described below, a key part of this analysis is to resolve the specific disruptions that would have the greatest influence on long-term TB burden.

Depending on its readiness, a country TB programme may take weeks or months to restore TB services to normal after a lockdown. This process may be delayed if, for example, laboratory capacity for diagnosis needs time to be reconstituted for $\mathrm{TB}$, or indeed if there 
remains a reluctance to seek care amongst those with TB symptoms, as a consequence of fear and stigma caused by the COVID-19 pandemic. Accordingly, to model the impact of the lockdown and its aftermath, we assumed two phases: a 'suspension' period of given duration, during which all impacts listed in Table 1 are in full effect, followed by a 'restoration' period, during which TB services are gradually (for simplicity, linearly) restored to normal. The suspension period is expected to last for as long as the lockdown is in effect, and potentially for longer: even once restrictions are eased, it may take some time for the reconstitution of TB services to begin, and for normal careseeking to begin resuming. We also assumed that TB transmission would revert to normal at the end of the suspension period, as a result of contact rates in the community rapidly being restored to normal. This assumption may be appropriate in high-burden, lowincome settings where physical distancing is less feasible than in high-income settings, but also where there are strong economic incentives to restore livelihoods as soon as possible. We present results for two disruption scenarios: a 'mild' scenario consisting of a 2-month suspension followed by a 2-month restoration period for TB services, and a 'severe' scenario consisting of a 3-month suspension followed by a 10 -month restoration period.

In each scenario we simulated the excess TB cases and deaths that would arise, over the period from 2020-2025, compared against a situation where TB services continue as normal over this period. In doing so, we ignore potential expansions in TB care in our comparator scenario, for example the scale-up of engagement with the private sector in India that was ongoing prior to the COVID-19 pandemic [21]. Our analysis therefore does not include the foregone benefits from continuing these expansions, and so we expect our model projections to be conservative with respect to the excess TB burden arising from the lockdown.
We also extrapolated estimates from our three focal countries to the global level using a simple approach, detailed in the supporting information (section 3). We used the India model to inform projections for countries with high TB burden and private sector involvement; the Kenya model to inform projections for countries where HIV is a driver of the TB epidemic; and the Ukraine model to inform projections for countries with a high proportion of drug-resistant TB, and hospital-based care delivery systems. Countries not in these groups were assigned the average impact of the three country models; we then aggregated country-level results to the global level.

\section{Sensitivity analysis}

We conducted a 'leave-one-out' analysis, in which we simulated the impact of the lockdown, but in the absence of a single element in Table 1 (for example, a scenario where all impacts are in full effect with the exception of diagnosis, which remains at pre-lockdown levels). This analysis allows an assessment of how excess TB burden may vary under more limited disruptions than the full set of scenarios identified in Table 1. In doing so, this analysis also helps to identify which types of disruption have the strongest contribution to excess TB burden. By performing a 'leave-one-out' simulation for each row of Table 1 in turn, we aimed to estimate the influence of each type of disruption.

Additionally, while many of the assumptions in Table 1 can be refined as further data become available, the effect of reduced contact rates in particular will be challenging to measure empirically. Recent analysis has examined the potential impact of different levels of transmission reduction [22], finding, for example, that these are unlikely to counterbalance the effects of disruptions in TB services. Our current analysis complements this work by capturing details of TB services. As discussed below, there is wide uncertainty around

Table 1

Expert consensus on potential disruptions arising from COVID-related lockdowns in three countries.

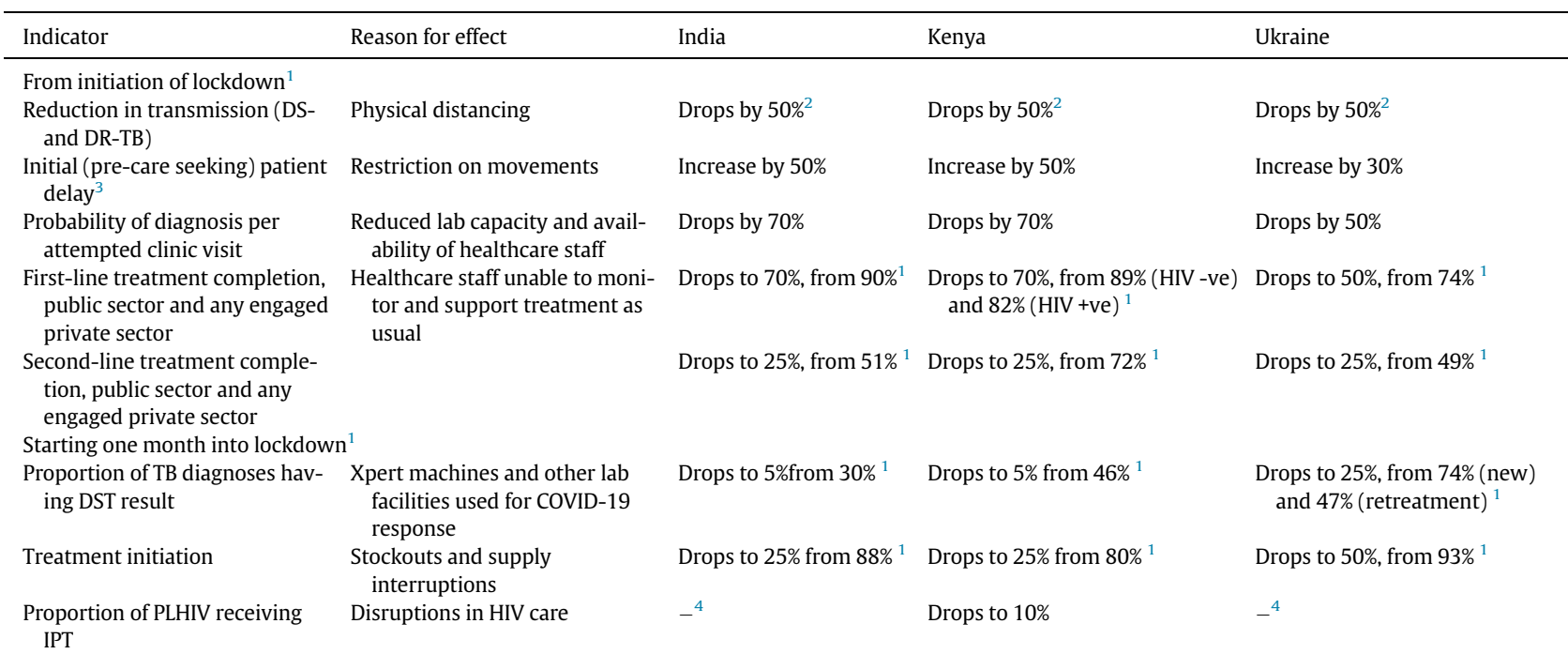

Footnotes: Scenarios were constructed through a rapid consultation with experts in the Stop TB Partnership and USAID, the former using information from a rapid survey of national TB programmes ${ }^{9}$. The scenarios listed here are not predictive, but illustrative on the basis of current information: they offer a basis for examining the potential impact of different types of disruption.

Footnotes:

Abbreviations: COVID-19: coronavirus disease 2019, DR: drug-resistant (i.e. rifampicin-resistant), DS: drug-susceptible, DST: drug susceptibility test, HIV: human immunodeficiency virus, IPT: isoniazid preventive therapy, PLHIV: people living with HIV, TB: tuberculosis.

1 For the initial levels and uncertainty intervals of these parameters in each country, see Tables S2-S5 (entries highlighted in yellow) in the supporting information.

2 Lockdowns would have the effect of reducing transmission in the community level, but also intensifying and prolonging exposure at the household level. As our models do not incorporate household vs community structure, these scenarios instead aim to capture the net effect of changes in household vs community transmission. In urban slums in particular, where TB transmission is strongest, overcrowding may tend to reduce the effect of any lockdown on community transmission. In section 3 in the supporting information, we present corresponding sensitivity analyses to these assumptions.

3 The initial patient delay is an assumed interval of active, infectious TB, prior to a patient's first presentation for care. It is calibrated to match epidemiological data (see Table S1 for data, and Tables S2-S5 for parameter estimates).

4 For simplicity, only the Kenya model incorporates the role of HIV/TB coinfection, which is estimated to account for $27 \%$ of incident TB. However, we note that Ukraine has a high burden of HIV as well; in the present study, our focus in Ukraine is on the role of drug-resistant TB. 
potential TB transmission reductions during a lockdown. There is evidence for contact rates being reduced by lockdown, even in informal settlements in Kenya [23], and masque use may further decrease transmission [24]. However, it is not yet known how strong these transmission-reducing effects might be for $\mathrm{TB}$, for example when weighed against the potential for prolonged and intensified exposure within the household. We therefore adopted a central estimate of $50 \%$ (see Table 1), while also adopting a wide range of scenarios from $10 \%$ to $75 \%$. Under each scenario we calculated the excess cases and deaths that would occur between 2020 and 2025.

\section{Role of the funding source}

SAM, AM, EM and SS are employed by the Stop TB Partnership, and SA is employed by USAID. The funders of the study otherwise had no role in study design, data collection, data analysis, data interpretation, or writing of the manuscript. The corresponding author had full access to all the data in the study and had final responsibility for the decision to submit for publication. All authors approved the final version of the manuscript submitted for publication.

\section{Results}

Figures S2 - S4, and Tables S2 - S5 in the supporting information, show the model calibrations to each of the targets shown in Table 1. On the basis of these calibrations, following a mild disruption we projected that between 2020 and 2025, in India there would be an increase of 182,000 TB cases (95\% Bayesian credible interval ( $\mathrm{CrI}$ ) 159-211 thousand) and 83,600 TB deaths (95\% CrI 77.5-90.6
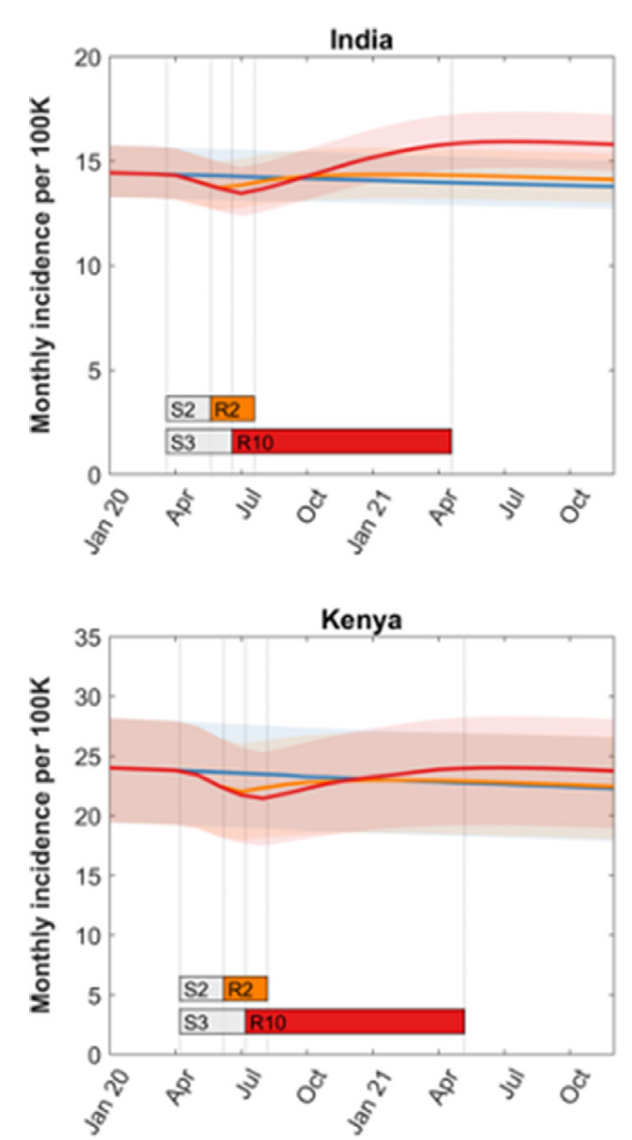

thousand). Likewise, in Kenya there would be an additional 489 cases (95\% CrI -2600-5700) and 2460 deaths (95\% CrI $1600-3800$ ), and in Ukraine an additional 840 cases (95\% CrI -460 - 1500) and 330 deaths (95\% CrI 153 - 570) (see Figs. 1 and 2, andTable 2). Although these results suggest the possibility of a net reduction in TB incidence in Kenya and Ukraine (as a result of the assumed 50\% reduction in transmission during the lockdown), such reductions only manifest under this mild scenario: in the case of a severe disruption, results suggest that TB incidence could increase by 3-9\% between 2020 and 2025 , and that TB deaths could increase by $4-16 \%$ in this same period.

In terms of the monthly dynamics, Figs. 1 and 2 illustrate that increases in incidence and mortality typically last longer than the duration of disruption. Indeed, in India, incidence was projected to remain at least $4 \%$ higher than a "business-as-usual" baseline for a period of 32 months, even in the mild scenario of a two-month suspension followed by two-month restoration (Fig. S5).

In India, the three specific service disruptions having the most effect on incidence and mortality are, in order: the increase in the initial patient delay before first presenting to a provider; the probability of diagnosis per visit to a provider; and the drop in treatment initiation (Fig. 3: leaving aside transmission, which we address below). Likewise in Kenya, the same three factors appear as most influential on the impact of the lockdown, on both TB incidence and mortality. In Ukraine, a setting with a high burden of drug resistance, the drop in second-line treatment completion was far more influential on overall impact, the drop in drug sensitivity testing, and the drop in the probability of $\mathrm{TB}$ diagnosis per visit to a provider were also important considerations.
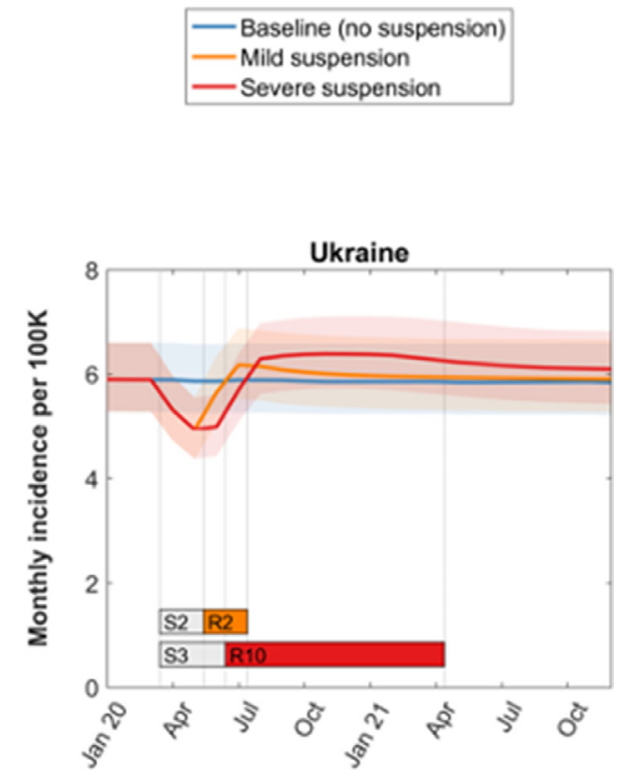

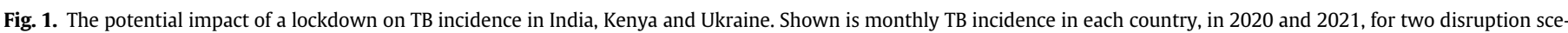

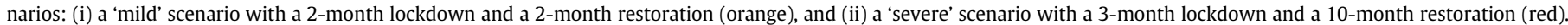

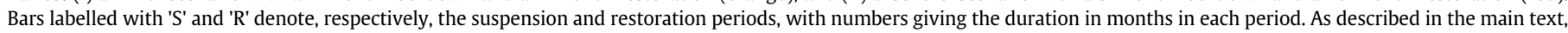

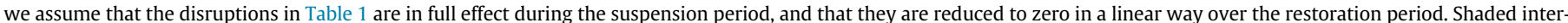

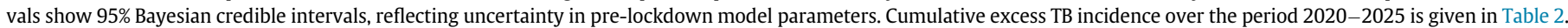



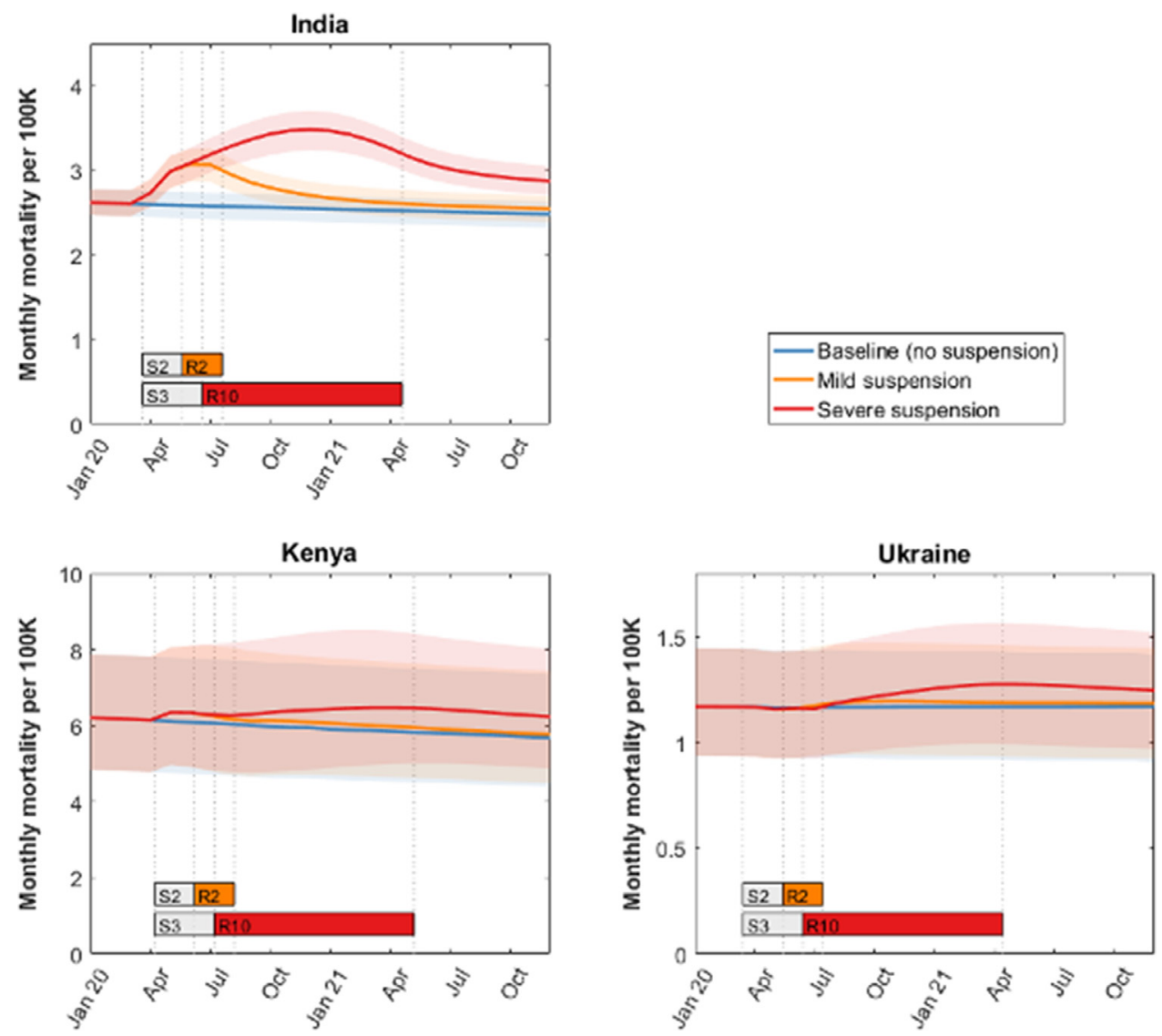

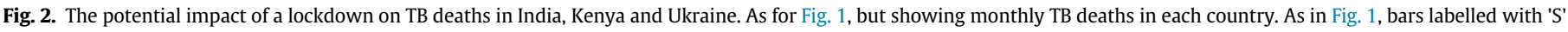

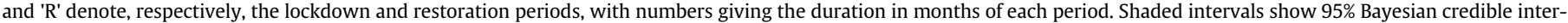
vals, reflecting uncertainty in pre-lockdown model parameters. Excess TB deaths over the period 2020-2025 are listed in Table 2.

Table 2

Excess TB incidence and deaths between 2020 and 2025 as a result of the different scenarios for COVID-related lockdowns.

\begin{tabular}{|c|c|c|c|c|}
\hline Country & $\begin{array}{l}\text { Excess cases between } 2020 \text { and } 2025 \\
\text { (\% increase) }[95 \% \mathrm{CrI}]\end{array}$ & & $\begin{array}{l}\text { Excess deaths between } 2020 \text { and } 2025 \\
\text { (\% increase) }[95 \% \mathrm{CrI}]\end{array}$ & \\
\hline & $\begin{array}{l}\text { 2-month suspension + 2-month } \\
\text { restoration }\end{array}$ & $\begin{array}{l}\text { 3-month suspension + 10-month } \\
\text { restoration }\end{array}$ & $\begin{array}{l}\text { 2-month suspension + 2-month } \\
\text { restoration }\end{array}$ & $\begin{array}{l}\text { 3-month suspension }+ \text {-month } \\
\text { restoration }\end{array}$ \\
\hline India & $\begin{array}{l}182,000[159,000-211,000] \\
(1.43 \%[1.30-1.65 \%])\end{array}$ & $\begin{array}{l}1190,000[1060,000-1330,000] \\
(9.25 \%[8.53-10.40 \%])\end{array}$ & $\begin{array}{l}83,600[77,500-90,600] \\
(3.65 \%[3.48-3.95 \%])\end{array}$ & $\begin{array}{l}361,000[333,000-394,000] \\
(15.70 \%[15-16.80 \%])\end{array}$ \\
\hline Kenya & $\begin{array}{l}489[-2660-5720] \\
(0.0076 \%[-0.35-0.81 \%])\end{array}$ & $\begin{array}{l}24,700[16,100-44,700] \\
(3.36 \%[2.28-6.11 \%])\end{array}$ & $\begin{array}{l}2460[1590-3840] \\
(1.30 \%[0.86-2.30 \%])\end{array}$ & $\begin{array}{l}12,500[8790-17,800] \\
(6.58 \%[5.30-10.0 \%])\end{array}$ \\
\hline Ukraine & $\begin{array}{l}835[-460-1520] \\
(0.60 \%[-0.30-1.03 \%])\end{array}$ & $\begin{array}{l}4350[826-6540] \\
(2.96 \%[0.52-4.47 \%])\end{array}$ & $\begin{array}{l}332[153-570] \\
(1.15 \%[0.55-1.73 \%])\end{array}$ & $\begin{array}{l}1340[815-1980] \\
(4.64 \%[2.84-6.27 \%])\end{array}$ \\
\hline
\end{tabular}

Abreviations: CrI-credible interval. All estimates are over the period from the beginning (1 Jan) of 2020 to the beginning of 2025 . Percentages show increases in cases and deaths relative to a baseline of no disruption (blue lines in Figs. 1,2).

The effect of disruptions in diagnosis, as well as in care-seeking and treatment initiation, is an expansion of the pool of individuals with undetected and untreated TB. Fig. 4 shows how the size of this pool grows over time; the right-hand panel illustrates the potential impact of a two-month campaign to reduce the prevalence of untreated TB in India through expanded case finding to reach an monthly notification target of 17 per 100,000 population per month, immediately upon easing of lockdown restrictions (i.e., implemented alongside the restoration of TB services). Depending on disruption severity and duration of restoration, such a two-month campaign could, pre-emptively, fully avert the adverse impact of the lockdown, by bringing 5-year incidence trends back to pre-lockdown levels. Below we discuss potential approaches for implementing these post-lockdown measures.
In addition to service disruptions, Fig. 3 illustrates that transmission reductions are also influential in the excess TB burden caused by a lockdown. We therefore conducted additional analyses to examine how model projections are affected by our assumptions for transmission. Fig. 5 shows estimates for excess incidence and mortality in all three countries, illustrating overall that, although it is possible for transmission reductions to give rise to net reductions in cumulative TB burden, this effect only occurs under the mildest disruption scenario (orange curve), and under the heaviest transmission reduction scenarios.

Additional analyses, provided in the supporting information (section 3 ), extrapolates from these three focal countries to the global level. This approach suggests, for example, that a severe suspension scenario could lead to an additional 4702,800 TB cases, and an additional 1044,800 TB deaths worldwide between 2020 and 2025 (Table S6). 

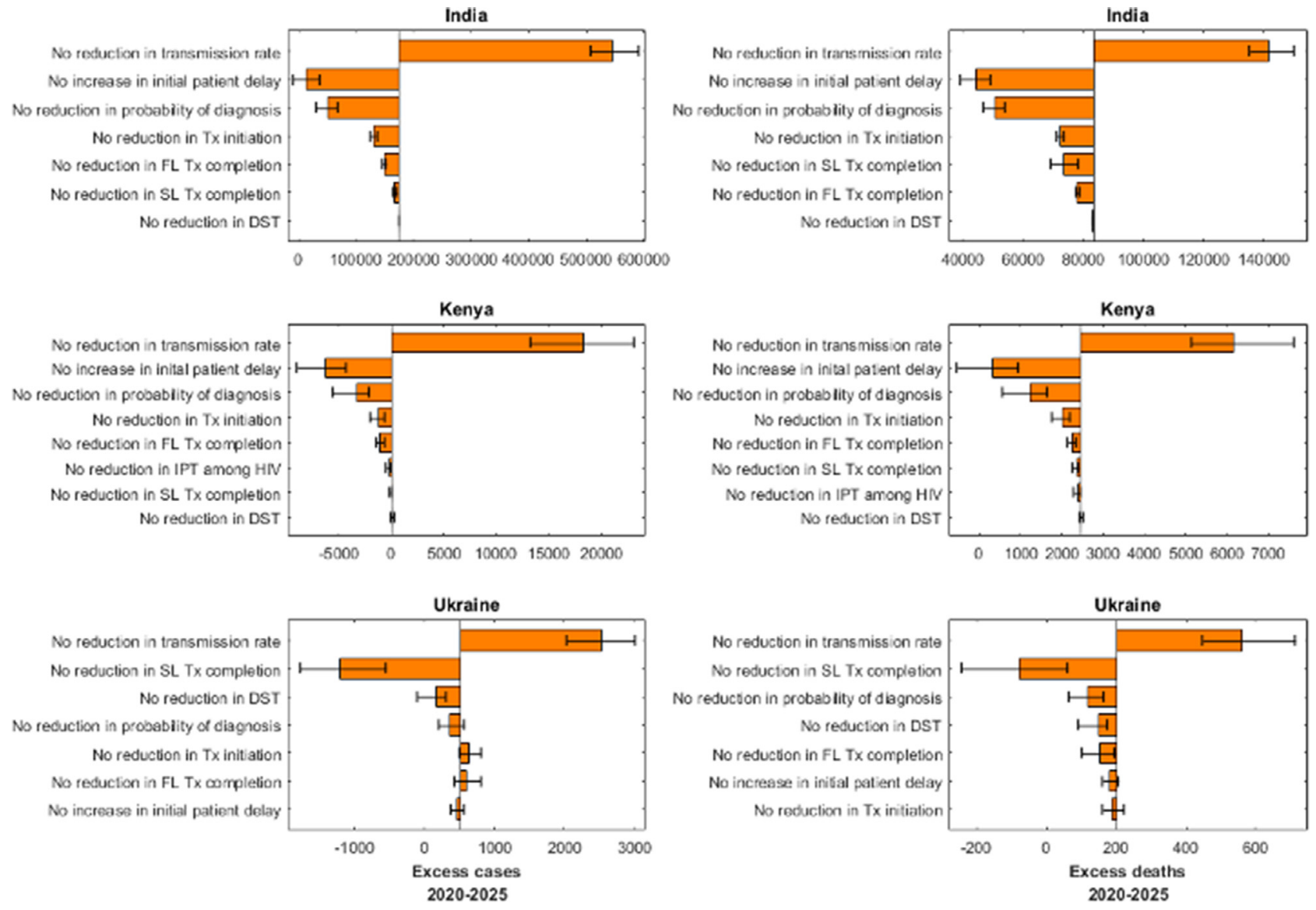

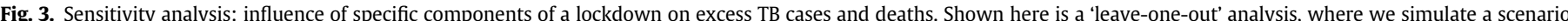

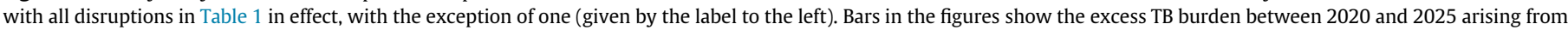

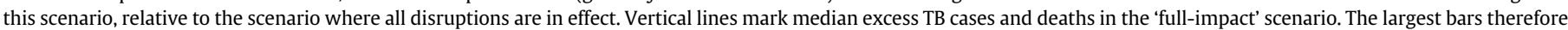

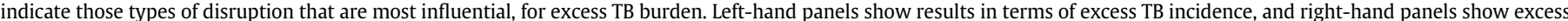

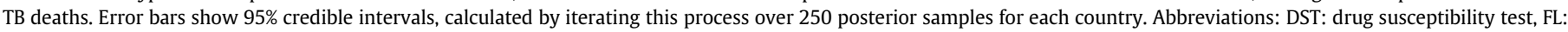
first-line, HIV: human immunodeficiency virus, IPT: isoniazid preventive therapy, SL: second-line, Tx: treatment.
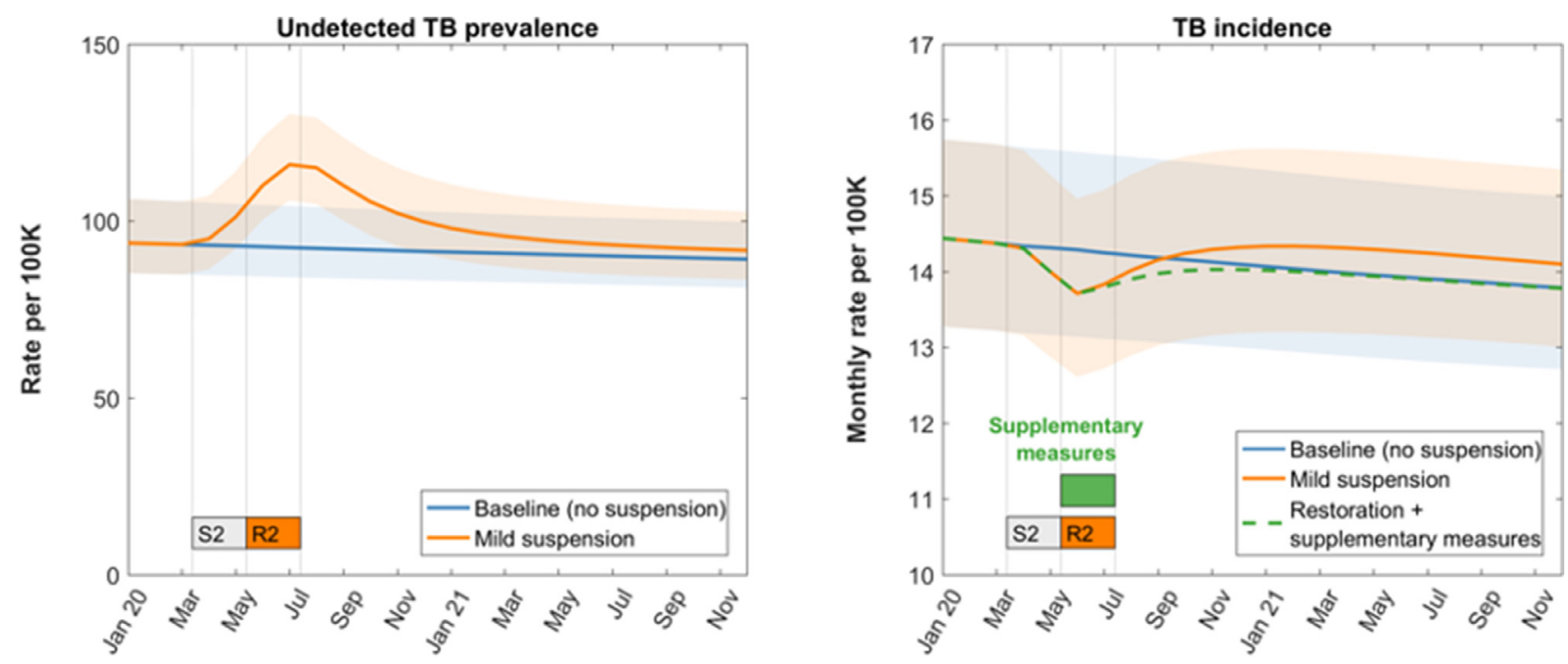

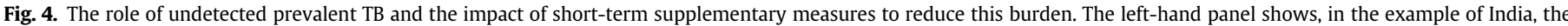

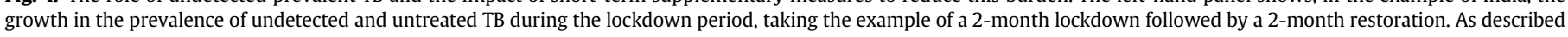

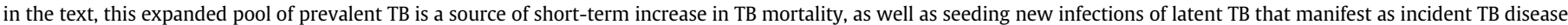

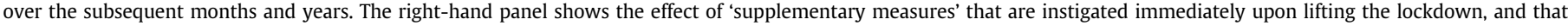

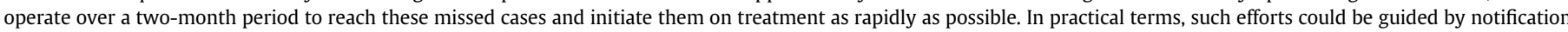

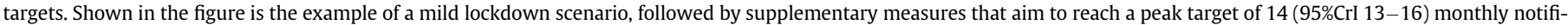
cations per 100,000 population. 

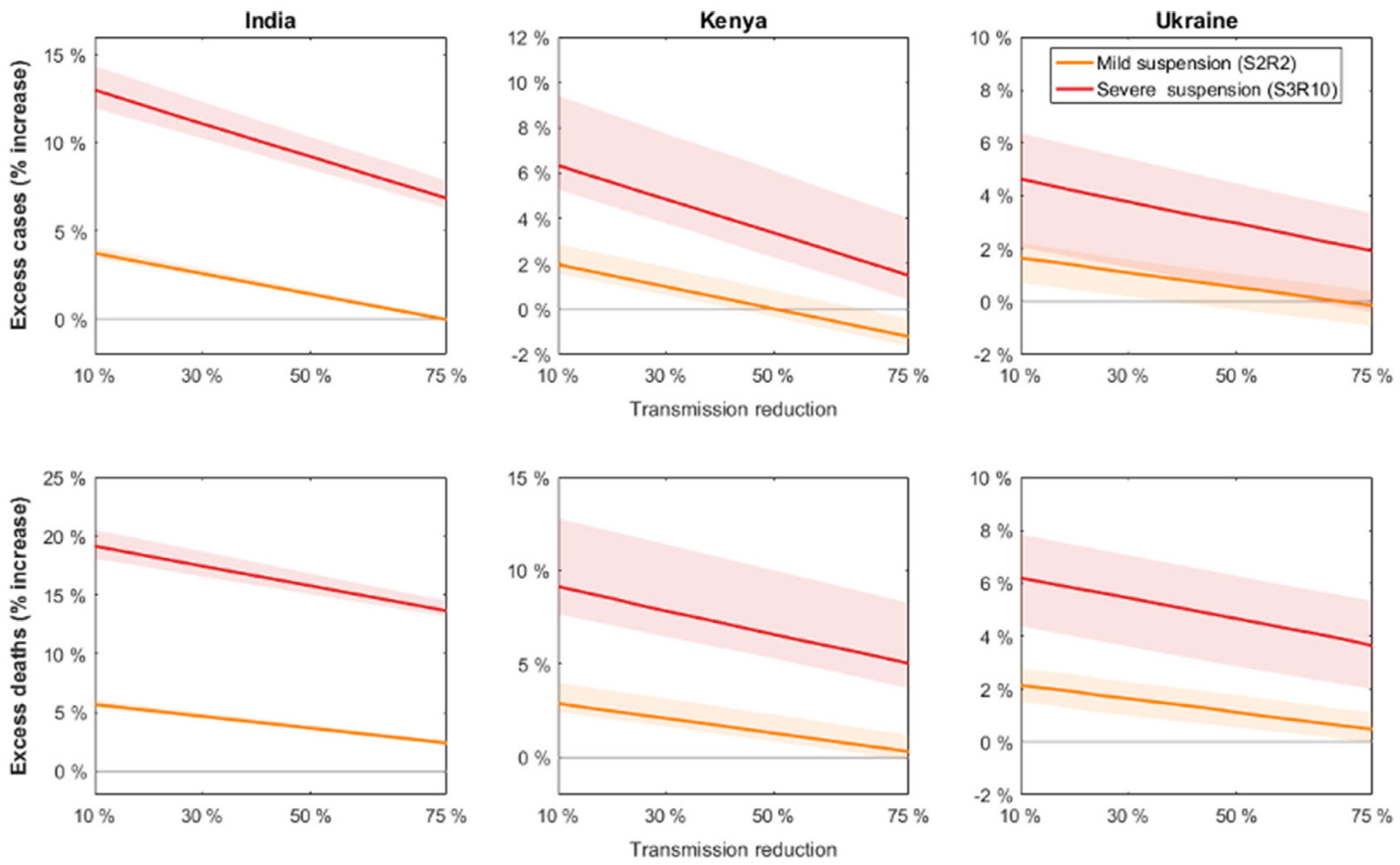

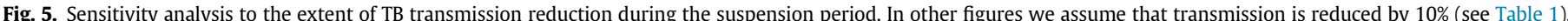

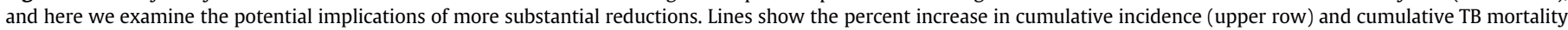

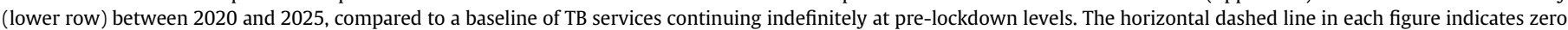

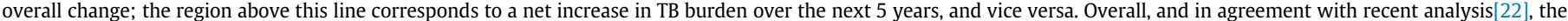

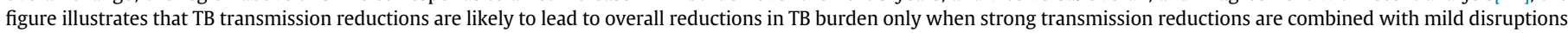
(orange lines, at $>50 \%$ transmission reductions for Kenya, and $>75 \%$ transmission reductions for India and Ukraine).

\section{Discussion}

This modelling analysis in three key countries illustrates that even short COVID-related lockdowns can generate long-lasting setbacks in TB control. Our results suggest that, in a severe disruption scenario, over the next five years TB deaths could see increases of $4-16 \%$, while TB incidence could see increases of $3-9 \%$, in the three countries studied here (Figs. 1 and 2, and Table 2). Our work also illustrates some differences between country settings, in the potential impact of lockdowns. For example, percentage increases in TB burden tend to be higher in India than in Kenya and Ukraine (Table 2). On the one hand, our estimates for the initial delay before first presentation for care are substantially higher for Kenya than for India (respectively, over a year and around 6 months respectively - see Tables S4 and S5). If the majority of transmission has already occurred by the time a symptomatic first presents for care, then service disruptions may have only limited impact on TB burden; we expect the same to apply in any high-prevalence setting, with prolonged pre-careseeking delays. On the other hand, Ukraine is a setting with substantially lower incidence than India or Kenya, and therefore weaker transmission: overall, we might expect lockdown-related increases in longterm incidence and mortality to be exacerbated in settings with higher levels of TB transmission, and vice-versa.

Our overall projections are broadly consistent with other, recent modelling work that also aimed to assess the potential impact of lockdown-related disruptions, on TB control [22,25]. However, our work complements these analyses by paying specific attention to the TB care cascade in different countries. While it is not possible to predict the trajectories of lockdowns, or associated disruptions in health services in any country, our work instead aims to identify the specific types of disruptions that would be most influential for long-term TB burden. These insights can be valuable in informing planning for post-lockdown TB measures in any country setting beyond those that we have examined here. In particular, Fig. 4 illustrates that the specific disruptions posing the greatest risk for long-term increases in TB incidence and mortality are those that permit an expansion in the pool of undetected, untreated TB: that is, disruptions hindering access to care amongst TB symptomatics, as well as the availability of diagnostic capacity when patients do access health faciities. Our analysis highlights how the expanded pool of undetected TB may continue to seed new infections of latent TB, many of which would take years to manifest as incident TB disease. Consequently, service disruptions give rise to a short-term escalation of TB mortality (Fig. 2), followed by a prolonged increase in incidence that could take years to undo (Fig. 1).

It follows that the adverse, long-term impact of these disruptions could be averted through focused efforts to address the problem of undetected $\mathrm{TB}$, immediately upon lifting restrictions. In Fig. 4 we illustrate one scenario, where such supplementary measures aim to reach a target of notifying 17 cases per 100,000 population, per month. We have not aimed here to define how such targets might be reached since optimal approaches are likely to vary across different settings. For example, it is likely that such supplementary measures would involve some form of active case-finding,[26,27] including contact tracing with longitudinal follow-up [28]. At the same time, in settings where careseeking rapidly restores to normal, it is possible that the manifestation of 'pent-up demand' could contribute substantially towards this target. In this scenario, it is important for TB programmes to retain the capacity needed to meet this post-lockdown surge in demand. In practice, a combination of both approaches may 
be needed, even at a time when TB programmes are operating under severe constraints in human and material resources. In the face of these constraints, anticipating the need for these concentrated efforts will be invaluable for planning, both by country programmes and by international donors who could support these efforts.

On the patient side, COVID-19 and pulmonary TB are both associated with respiratory symptoms. If, during the current pandemic, COVID-19 comes to be seen as a "TB-like" disease, public recognition of the importance of recognising TB symptoms may wane once COVID-19 is thought to be under control. Additional efforts may therefore be needed to address these misperceptions. An additional concern is that COVID-19 may carry stigma in many communities, and this stigma may transfer to individuals with TB as well [29]. Conversely, there may be opportunities to leverage synergies between the two diseases; for example, integrated TB and COVID-19 screening and testing algorithms or combined contact investigation strategies. Any such strategies based on respiratory symptoms could use similar infrastructure and staff to mitigate both the direct impacts of SARSCoV-2 transmission and the indirect effects of augmented $M$. tuberculosis transmission. We emphasise that, readiness to restore TB services as rapidly as possible, combined with focused efforts to 'catch up' on missed diagnoses, will be critical in limiting any long-term setback to TB care efforts as a result of the COVID-19 response.

One important uncertainty is the potential impact of the lockdown, on TB transmission. Assuming a 50\% reduction in transmission, our results suggest the possibility of a mild disruption to yield a net reduction in 5-year incidence, in Kenya and Ukraine (Fig.1, and Table 2). More broadly, our sensitivity analysis (Fig. 5) illustrates that transmission reductions would only lead to a net decrease in TB burden over the next five years if these reductions are relatively strong, coupled with disruptions to TB services that are relatively mild. These results are in agreement with recent modelling analysis, that similarly showed how transmission reductions are unlikely to outweigh the negative impacts of disruptions to TB services [22]. We caution that - as with any models not fully capturing household and other population structures - these simulations are likely to overestimate the rate-of-change in TB incidence over short timescales, such as the drop in TB incidence shown during the lockdown period. Nonetheless, these and similar results remain qualitatively illustrative of the potential importance of changes in transmission.

A key implication of our scenario analysis is the centrality of establishing surveillance and other data systems to inform the extent of lockdown-associated disruptions in TB care. For example, TB notifications (e.g., ref [30]) can be monitored in real time at a national and subnational level, to evaluate the depth and duration of any reductions in TB diagnosis at different stages of any lockdown. If these indicators suggest persistent declines in notifications and/or falling levels of treatment success, targeted interventions (e.g., active case finding, treatment support, or expanded access) can be rapidly implemented. As contact investigation for TB is implemented, surveillance of infection and active TB can be established and time trends can be used to inform whether household transmission has increased and/or access to care has declined, again at the local, subnational, and national levels. In the longer term, community-based surveys (e.g., serial surveys of TB infection in young children [31], can be conducted to explore the impact of lockdowns on TB transmission more broadly.

We note that the present analysis does not address the potential for direct interactions between TB and COVID-19 (for example, increased risk of COVID-19 mortality amongst individuals with TB). For this reason, our estimates for excess mortality in particular are likely to be conservative. For example, early evidence suggests that existing TB infection, whether latent or active, can be a strong risk factor for severe disease resulting from SARS-CoV-2 infection[32], although this association may vary by setting [33,34]. Moreover, through pre-existing lung damage [35], past TB infection might also predispose individuals to poorer outcomes from COVID-19. Further evidence on both potential impacts, particularly from longitudinal cohorts) [36], would be invaluable for future work examining these potential pathogen-pathogen interactions.

As with any modelling study, our analysis involves several simplifications. Our models do not distinguish age structure, nor pulmonary versus extrapulmonary TB, instead taking an average over these distinctions. For our modelling of Kenya, for simplicity we have only captured the transmission dynamics of TB, treating HIV incidence as pre-specified. Our model therefore does not capture the potential TB implications of disruptions in HIV care, and for this reason may be conservative. Lockdowns are likely to reduce community transmission but at the expense of intensifying and prolonging household and congregate setting exposure. Faithfully capturing household contact structure is generally not feasible in compartmental models such as in the current analysis, and instead we have taken a simple approach of an assumed overall net reduction in transmission. Finally, while our work has concentrated on three country settings capturing key challenges in the TB care cascade, future work should address other settings (such as in South America), as well as the effect of disruptions at the subnational level, if lockdowns against COVID-19 increasingly become implemented at city- and provincial-levels.

In conclusion, our analysis illustrates how increases in TB burden can take months to manifest, but years to undo. Even if a lockdown is a period of curtailed programmatic activity, our results also highlight how this period might be used by country programmes and international agencies to prepare for the timely restoration of TB control activities and even "catch-up" campaigns upon easing of restrictions, to prevent such long-term negative impacts from taking hold. The resilience of systems to end TB worldwide will depend critically on readiness to restore, supplement and monitor TB services as rapidly as possible.

\section{Data sharing}

All data used and generated in this study is available in the manuscript and supporting information.

\section{Declaration of competing interest}

SA is employed by USAID and SAN, AM, EM, and SS are employed by the Stop TB Partnership. The other authors declare no conflicts of interest.

\section{Funding}

This work was supported by the Stop TB Partnership, and by USAID. NA gratefully acknowledges additional support from the Bill and Melinda Gates Foundation, and the UK Medical Research Council.

\section{Author contributions}

SS, SA, and NA conceived the study, and NA, DD, and CP designed the approach. SA, SAN, AM, EM, and SS provided expert input in constructing the model assumptions, and validated model findings. LC, $\mathrm{HF}$, JFV, and CP performed the analysis, and all authors contributed to the interpretation. LC, HF, NA, and DD wrote a first draft of the manuscript, and all authors contributed to the final version.

\section{Acknowledgements}

We gratefully acknowledge support from Sara Gonzalez Andino and Shinichi Takenaka from Stop TB Partnership, in the process of development of modelling assumptions. 


\section{Supplementary materials}

Supplementary material associated with this article can be found, in the online version, at doi:10.1016/j.eclinm.2020.100603.

\section{References}

[1] Anderson RM, Heesterbeek H, Klinkenberg D, Hollingsworth TD. How will country-based mitigation measures influence the course of the COVID-19 epidemic? Lancet 2020 .

[2] Flaxman S, Mishra S, Gandy A, et al. Estimating the number of infections and the impact of non-pharmaceutical interventions on COVID-19 in 11 European countries. Imp Coll Lond 2020.

[3] Prem K, Liu Y, Russell TW, et al. The effect of control strategies to reduce social mixing on outcomes of the COVID-19 epidemic in Wuhan, China: a modelling study. Lancet Public Heal 2020.

[4] Walker PGT, White MT, Griffin JT, Reynolds A, Ferguson NM, Ghani AC. Malaria morbidity and mortality in Ebola-affected countries caused by decreased healthcare capacity, and the potential effect of mitigation strategies: a modelling analysis. Lancet Infect Dis 2015.

[5] Arinaminpathy N, Dye C. Health in financial crises: economic recession and tuberculosis in Central and Eastern Europe. J R Soc Interface 2010;7.

[6] Parpia AS, Ndeffo-Mbah ML, Wenzel NS, Galvani AP. Effects of response to 2014 2015 ebola outbreak on deaths from malaria, HIV/AIDS, and tuberculosis, West Africa. Emerg Infect Dis 2016.

[7] World Health Organization. Global tuberculosis report 2019. World Health Organization; 2019 https://www.who.int/tb/publications/global_report/en/ (accessed Jan 13, 2020).

[8] Raviglione M, Sulis G. Tuberculosis 2015: burden, challenges and strategy for control and elimination. Infect Dis Rep 2016;8:6570.

[9] Stop TB Partnership. we did a rapid assessment: the TB response is heavily impacted by the COVID-19 pandemic. 2020. http://stoptb.org/news/stories/2020/ ns20_014.html.

[10] Ismail N, Moultrie H. Impact of COVID-19 intervention on TB testing in South Africa. Natl. Inst. Commun. Dis. 2020 https://www.nicd.ac.za/wp-content/ uploads/2020/05/Impact-of-Covid-19-interventions-on-TB-testing-in-SouthAfrica-10-May-2020.pdf.

[11] Reid MJA, Arinaminpathy N, Bloom A, et al. Building a tuberculosis-free world: the Lancet Commission on tuberculosis. Lancet 2019:393:1331-84.

[12] Vesga JF, Hallett TB, Reid MJA, et al. Assessing tuberculosis control priorities in high-burden settings: a modelling approach. Lancet Glob Heal 2019 published online March.

[13] Arinaminpathy N, Batra D, Khaparde S, et al. The number of privately treated tuberculosis cases in India: an estimation from drug sales data. Lancet Infect Dis 2016;16.

[14] Enos M, Sitienei J, Ong’ang’o J, et al. Kenya tuberculosis prevalence survey 2016: challenges and opportunities of ending TB in Kenya. PLoS ONE 2018.

[15] Pavlenko E, Barbova A, Hovhannesyan A, et al. Alarming levels of multidrug-resistant tuberculosis in Ukraine: results from the first national survey. Int. J. Tuberc Lung Dis. 2018.
[16] Poole D, Raftery AE. Inference for deterministic simulation models: the Bayesian Melding approach. J Am Stat Assoc 2000;95:1244

[17] Alkema L, Raftery AE, Brown T. Bayesian melding for estimating uncertainty in national HIV prevalence estimates. Sex Transm Infect 2008;84:i11-6.

[18] Menzies NA, Cohen T, Lin H-H, Murray M, Salomon JA. Population health impact and cost-effectiveness of tuberculosis diagnosis with Xpert MTB/RIF: a dynamic simulation and economic evaluation. PLoS Med 2012;9:e1001347.

[19] WHO Regional Office for Europe. Rapid communication on the role of the GeneXpert $^{\circledR}$ platform for rapid molecular testing for SARS-CoV-2 in the WHO European Region. 2020.

[20] Adepoju P. Tuberculosis and HIV responses threatened by COVID-19. Lancet HIV 2020.

[21] JEET Consortium. Joint effort for elimination of tuberculosis. http://mospi.nic.in/ sites/default/files/publication_reports/nss_rep574.pdf (accessed Dec 20, 2018).

[22] McQuaid CF, McCreesh N, Read JM, et al. The potential impact of COVID-19related disruption on tuberculosis burden. Eur Respir J 2020.

[23] Quaife M, van Zandvoort K, Gimma A, et al. The impact of COVID-19 control measures on social contacts and transmission in Kenyan informal settlements. medRxiv 2020 2020.06.06.20122689.

[24] Dharmadhikari AS, Mphahlele M, Stoltz A, et al. Surgical face masks worn by patients with multidrug-resistant tuberculosis. Am J Respir Crit Care Med 2012.

[25] Glaziou P. Predicted impact of the COVID-19 pandemic on global tuberculosis deaths in 2020. medRxiv 2020 2020;04:20079582 28.

[26] Yuen CM, Amanullah F, Dharmadhikari A, et al. Turning off the tap: stopping tuberculosis transmission through active case-finding and prompt effective treatment. Lancet. 2015 .

[27] Azman AS, Golub JE, Dowdy DW. How much is tuberculosis screening worth? estimating the value of active case finding for tuberculosis in South Africa, China, and Indi. BMC Med 2014

[28] Fox GJ, Nhung N V, Sy DN, et al. Household-contact investigation for detection of tuberculosis in Vietnam. N Engl J Med 2018;378:221-9.

[29] World Health Organization. Social stigma associated with COVID-19. 2020

[30] Central TB Division India. Nikshay dashboard. 2020. https://reports.nikshay.in/ Reports/TBNotification.

[31] Middelkoop K, Bekker LG, Morrow C, Lee N, Wood R. Decreasing household contribution to TB transmission with age: a retrospective geographic analysis of young people in a South African township. BMC Infect Dis 2014.

[32] Liu Y, Bi L, Chen Y, et al. Active or latent tuberculosis increases susceptibility to COVID-19 and disease severity. medRxiv 2020.

[33] Stochino C, Villa S, Zucchi P, Parravicini P, Gori A, Raviglione MC. Clinical characteristics of COVID-19 and active tuberculosis co-infection in an Italian reference hospital. Eur Respir J 20202001708.

[34] Motta I, Centis R, D’Ambrosio L, et al. Tuberculosis, COVID-19 and migrants: preliminary analysis of deaths occurring in 69 patients from two cohorts. Pulmonology 2020;26:233-40.

[35] Ravimohan S, Kornfeld H, Weissman D, Bisson GP. Tuberculosis and lung damage: from epidemiology to pathophysiology. Eur. Respir. Rev. 2018.

[36] Tadolini M, Codecasa LR, García-García J-M, et al. Active tuberculosis, sequelae and COVID-19 co-infection: first cohort of 49 cases. Eur Respir J 2020:2001398. 